\title{
Stability and semiclassics in self-generated fields
}

\author{
László Erdős * \\ Institute of Mathematics, University of Munich \\ Theresienstr. 39, D-80333 Munich, Germany \\ Søren Fournais ${ }^{\dagger}$ \\ Department of Mathematical Sciences, Aarhus University \\ Ny Munkegade 118, DK-8000 Aarhus, Denmark \\ and \\ Jan Philip Solovej $\ddagger$ \\ Department of Mathematics, University of Copenhagen \\ Universitetsparken 5, DK-2100 Copenhagen, Denmark
}

Oct 18, 2011

\begin{abstract}
We consider non-interacting particles subject to a fixed external potential $V$ and a self-generated magnetic field $B$. The total energy includes the field energy $\beta \int B^{2}$ and we minimize over all particle states and magnetic fields. In the case of spin- $1 / 2$ particles this minimization leads to the coupled Maxwell-Pauli system. The parameter $\beta$ tunes the coupling strength between the field and the particles and it effectively determines the strength of the field. We investigate the stability and the semiclassical asymptotics, $h \rightarrow 0$, of the total ground state energy $E(\beta, h, V)$. The relevant parameter measuring the field strength in the semiclassical limit is $\kappa=\beta h$. We are not able to give the exact leading order semiclassical asymptotics uniformly in $\kappa$ or even for fixed $\kappa$. We do however give upper and lower bounds on $E$ with almost matching dependence on $\kappa$. In the simultaneous limit $h \rightarrow 0$ and $\kappa \rightarrow \infty$ we show that the standard non-magnetic Weyl asymptotics holds. The same result also holds for the spinless case, i.e. where the Pauli operator is replaced by the Schrödinger operator.
\end{abstract}

\footnotetext{
*Partially supported by SFB-TR12 of the German Science Foundation. lerdos@math.lmu.de

${ }^{\dagger}$ Work partially supported by the Lundbeck Foundation, the Danish Natural Science Research Council and the European Research Council under the European Community's Seventh Framework Program (FP7/20072013)/ERC grant agreement 202859. fournais@imf.au.dk

${ }^{\ddagger}$ Work partially supported by the Danish Natural Science Research Council and by a Mercator Guest Professorship from the German Science Foundation. solovej@math.ku.dk
} 
AMS 2010 Subject Classification: 35P15, 81Q10, 81Q20

Key words: Semiclassical eigenvalue estimate, Maxwell-Pauli system, Scott correction, Running title: Stability and semiclassics with self-generated field.

\section{Introduction}

An important problem in spectral analysis is to determine or bound the sum of the negative eigenvalues of a Schrödinger operator $-\Delta-V(x)$, i.e.,

$$
\operatorname{Tr}(-\Delta-V(x))_{-}
$$

under appropriate conditions on the potential $V$. We use the convention that $x_{-}=(x)_{-}=$ $\min \{x, 0\}$ when $x$ is either a real number or a self-adjoint operator. This problem is of particular interest in quantum mechanics as it gives the ground state energy of a gas of free fermions moving in the exterior potential $V$.

A generalization of this problem is to consider not only a potential $V$ but also an exterior magnetic field given by the vector potential $A$. The corresponding magnetic Schrödinger operator is $(-i \nabla+A)^{2}-V(x)$ acting in $L^{2}\left(\mathbb{R}^{d}\right)$. A further generalization is to consider the particles as having spin $-\frac{1}{2}$ and introduce the magnetic Pauli operator $[\boldsymbol{\sigma} \cdot(-i \nabla+A)]^{2}-V$, where in $d=3$ dimensions $\boldsymbol{\sigma}=\left(\sigma_{1}, \sigma_{2}, \sigma_{3}\right)$ denotes the vector of $2 \times 2$ Pauli matrices. The Pauli operator acts in $L^{2}\left(\mathbb{R}^{3} ; \mathbb{C}^{2}\right)$. Much work has gone into understanding the semiclassical asymptotics of the sum of negative eigenvalues, i.e., the asymptotics for small $h>0$ of

$$
\operatorname{Tr}\left((-i h \nabla+A)^{2}-V(x)\right)_{-} \quad \text { or } \operatorname{Tr}\left([\boldsymbol{\sigma} \cdot(-i h \nabla+A)]^{2}-V(x)\right)_{-} .
$$

It is well known that under appropriate conditions on $A$ and $V$ the leading behavior as $h$ tends to zero is given by the Weyl formulas

$$
(2 \pi h)^{-d} \iint_{\mathbb{R}^{d} \times \mathbb{R}^{d}}\left(p^{2}-V(x)\right)_{-} \mathrm{d} x \mathrm{~d} p \quad \text { or } \quad 2(2 \pi h)^{-3} \iint_{\mathbb{R}^{3} \times \mathbb{R}^{3}}\left(p^{2}-V(x)\right) \_\mathrm{d} x \mathrm{~d} p,
$$

respectively. Here the factor of 2 on the second integral is due to the spin degrees of freedom in the Pauli operator, i.e., the fact that it is a $2 \times 2$-matrix valued operator. Note that the limiting semiclassical behavior is non-magnetic, i.e. fixed magnetic fields do not influence the leading order semiclassics. For simplicity we will consider the $d=3$ dimensional case only and we denote the Schrödinger operator $T_{h}^{\mathrm{S}}(A)=(-i h \nabla+A)^{2}$ and the Pauli operator $T_{h}^{\mathrm{P}}(A)=[\boldsymbol{\sigma} \cdot(-i h \nabla+A)]^{2}$. The magnetic field is $B=\nabla \times A$.

In this paper we will address a related and equally important issue, namely the case when the magnetic field is not a fixed external field, but the self-generated classical magnetic field generated by the particles themselves. 
We will consider the external potential $V$ to be a fixed (given) function in $\mathbb{R}^{3}$, we assume $V \in L_{l o c}^{1}\left(\mathbb{R}^{3}\right)$ and we will always work with the flat Euclidean metric. The vector potential $A$ will be optimized to minimize the total energy consisting of the energy of the particles and the field energy

$$
\int B^{2}=\int|\nabla \times A|^{2}
$$

(we use the convention that unspecified integrals are always on $\mathbb{R}^{3}$ w.r.t. the Lebesgue measure). The problem we consider is thus to determine the energy

$$
E^{\mathrm{S}, \mathrm{P}}(\beta, h, V)=\inf _{A}\left[\operatorname{Tr}\left(T_{h}^{\mathrm{S}, \mathrm{P}}(A)-V\right)_{-}+\beta \int|\nabla \times A|^{2}\right]
$$

for $\beta, h>0$, where the infimum runs over all vector fields $A \in H^{1}\left(\mathbb{R}^{3} ; \mathbb{R}^{3}\right)$; in fact minimizing only for all $A \in C_{0}^{\infty}\left(\mathbb{R}^{3} ; \mathbb{R}^{3}\right)$ gives the same infimum. See Appendix $\mathrm{A}$ for a discussion of equivalent variational spaces for this energy and for the precise definition of the operator $T_{h}^{\mathrm{S}, \mathrm{P}}(A)-V$ and the sum of its negative eigenvalues. We will omit the superscripts $\mathrm{S}, \mathrm{P}$, when making general statements valid for both the Schrödinger and Pauli cases.

Here $\beta$ is an additional parameter setting the strength of the coupling of the particles to the field. In a given physical system the values of $h$ and $\beta$ are given, but as is standard in semiclassical analysis we leave them as free parameters. Formally $\beta=\infty$ corresponds to the non-magnetic case; smaller $\beta$ means that a larger effect of the magnetic field is expected.

The Euler-Lagrange equation corresponding to the variational problem (10) above is

$$
\beta \nabla \times B=J_{A}
$$

where $J_{A}$ is the current of the Fermi gas, which in the Schrödinger case is

$$
J_{A}(x)=-\operatorname{Re}\left[(-i h \nabla+A) 1_{(-\infty, 0]}\left(T_{h}(A)-V\right)\right](x, x)
$$

and in the Pauli case is

$$
J_{A}(x)=-\operatorname{Re}\left[\operatorname{Tr} \mathbb{C}^{2}\left(\boldsymbol{\sigma}(\boldsymbol{\sigma} \cdot(-i h \nabla+A)) 1_{(-\infty, 0]}\left(T_{h}(A)-V\right)\right)\right](x, x) .
$$

In other words the Euler-Lagrange equations are the non-linear coupled (time independent) Maxwell-Schrödinger or Maxwell-Pauli equations where we deliberately ignored Gauss' equation for $V$ in order to obtain a general result. In the application to large atoms discussed below we will consider the special case when $V$ solves Gauss' equation.

There are three natural questions about the energy $E(\beta, h, V)$. First of all we may ask whether the energy is finite, i.e., not negative infinity. We refer to this as stability. In Theorem 2.1 we give bounds on the energy in the Pauli case under essentially sharp assumptions on $V$. The corresponding results for the Schrödinger case are well known and are also discussed in Section 2 . 
The second natural question is whether the inclusion of the self-generated magnetic field will actually lower the energy at all. For both the Pauli and the Schrödinger cases this is indeed true (see Appendix (B), i.e., there exist potentials $V$ and parameters $\beta, h$ (not necessarily the same for the Pauli and Schrödinger cases) such that

$$
E(\beta, h, V)<E(\beta=\infty, h, V)
$$

The third and, actually, main question we address is how the inclusion of the magnetic field influences the semiclassical asymptotics $(h \rightarrow 0)$ for the sum of the negative eigenvalues. Standard semiclassical results typically assume that the physical data (external potential, vector potential, metric etc.) are smooth. If as above these data arise as self-generated and thus determined internally via a variational principle the smoothness is not a-priori given.

In Theorem 2.2 we establish under the appropriate assumptions on $V$ that the semiclassical, i.e., $h \rightarrow 0$, asymptotics of $E(\beta, h, V)$ in the case when $\beta h \rightarrow \infty$ simultaneously with $h \rightarrow 0$, is given by the standard non-magnetic Weyl formula. The case of large $\beta h$ is the case of greatest physical interest as the magnetic field in general gives rise to a small effect. In [EFS2] we give improved estimates on the error term to the non-magnetic Weyl term.

It is however at least of mathematical interest to understand the behavior of $E(\beta, h, V)$ also when $\beta h$ is smaller, i.e., when the effect of the magnetic field is greater. Unfortunately, we have not been able to establish the exact semiclassical asymptotics in this case. We do show however in Theorem 2.1 again under appropriate assumptions on $V$ that the Pauli energy $h^{3} E^{\mathrm{P}}(\beta, h, V)$ is bounded from above and below by functions of $\kappa:=\beta h$. These functions have almost matching asymptotics in the regime of small $\beta h$. In proving the upper bound we rely heavily on the construction of zero-modes in [ES1]. The similar result for the Schrödinger case is well-known and, in fact, here the bounds do not depend on $\kappa$ (see (66) below).

Theorem 2.1. moreover shows that for sufficiently small $\kappa=\beta h$ (depending on $V$ ) the magnetic and non-magnetic energies are different, i.e., (3) holds, since the former is independent of $\kappa$, while the latter scales at least as $\kappa^{-3+\varepsilon}$ for any $\varepsilon>0$. In summary, we have established that $\beta \sim h^{-1}$ is the correct threshold for the Pauli operator to observe the effect of the magnetic field in the leading order semiclassics.

Our results will be stated and proved for the Pauli operator and we will remark the modifications for the Schrödinger case.

\subsection{Applications to large atoms}

One of the main applications of precise semiclassical estimates is to investigate the ground state energy of large atoms and molecules. It is a celebrated result of Lieb and Simon LS] (see also $[\mathrm{L}]$ ) that the energy of a neutral atom or molecule with nuclear charge $Z$ obeys the Thomas-Fermi asymptotics, - (const.) $Z^{7 / 3}$, in the large $Z$ limit. The subleading correction to order $Z^{2}$ is known as the Scott correction and was established for atoms in [H, SW1] and for molecules in [IS] (see also [SW2, SW3, SS]). The next term in the expansion of order $Z^{5 / 3}$ 
was rigorously established for atoms in [FS]. The large $Z$ asymptotics can be viewed as a semiclassical limit with $h=Z^{-1 / 3}$ being the semiclassical parameter. The Scott term thus corresponds to the next order semiclassical estimate. In the results mentioned so far on the large $Z$ asymptotics, magnetic interactions are ignored.

Magnetic fields in this context were first taken into account as external fields, either a homogeneous one [Y, LSY1, LSY2, (see also [Sob1, Sob2, Sob3 for improved semiclassical estimates and [Iv1, Iv2] for inclusion of the Scott correction) or an inhomogeneous one [ES1] but subject to certain regularity conditions. Self-generated magnetic fields, obtained from Maxwell's equation (2) are not known to satisfy these conditions. In [ES3 the validity of Thomas-Fermi theory was extended by allowing a self-generated magnetic field that interacts with the electrons. This means that the focus was on the absolute ground state of the system, after minimizing for both the electron wave function and for the magnetic field. Without going into details we mention that the Thomas-Fermi theory can be viewed as the semiclassical approximation to our Maxwell-Pauli system but with the Gauss' equation included. It was shown that the additional magnetic field does not change the leading order Thomas-Fermi energy. This holds if $Z \alpha^{2}$ is sufficiently small, where $\alpha$ is the fine structure constant; for large values of $Z \alpha^{2}$ the system is unstable (see [ES3] for more details).

The semiclassical problem corresponding to a self-generated magnetic field is exactly of the type (1) we discuss in the present paper for $\beta h \rightarrow \infty$. In order to establish the Scott correction for self-generated magnetic fields, it is necessary to establish the semiclassical expansion of $E(\beta, h, V)$ up to subleading order if $\beta h^{2}$ is bounded from below. Such an improved semiclassical estimate is proven in a separate paper [EFS2] and the Scott term asymptotics is proved in EFS3.

Acknowledgment: JPS thanks R. Seiringer and C. Hainzl for fruitful discussions.

\section{Results}

In this section we will state and discuss our two main theorems. The proofs are given in the following sections.

Theorem 2.1 (Stability bounds for the Pauli energy). Assuming $V \in L_{l o c}^{1}\left(\mathbb{R}^{3}\right)$ and $[V]_{+} \in$ $L^{5 / 2}\left(\mathbb{R}^{3}\right) \cap L^{4}\left(\mathbb{R}^{3}\right)$, we have, for the Pauli operator

$$
h^{3} E^{\mathrm{P}}(\beta, h, V) \geq-C \int[V]_{+}^{5 / 2}-C(\beta h)^{-3} \int[V]_{+}^{4} .
$$

for $C>0$ and all $h \in(0, \infty)$ and $\beta \in(0, \infty]$. On the other hand if $V \in C_{0}^{1}\left(\mathbb{R}^{3}\right)$ then for all $0<\varepsilon<1 / 3, h \in(0, \infty)$ and $\beta \in(0, \infty]$

$$
h^{3} E^{\mathrm{P}}(\beta, h, V) \leq-C^{\prime} \int[V]_{+}^{5 / 2}-C_{\varepsilon}(\beta h)^{-3+2 \varepsilon} \int[V]_{+}^{4-\varepsilon}+\mathcal{E}_{V}(h)
$$


for positive constants $C^{\prime}$ and $C_{\varepsilon}$, the latter depending on $\varepsilon$, and with an error function $h \mapsto$ $\mathcal{E}_{V}(h)$, depending only on $\varepsilon, h$, and $V$ and satisfying $\lim _{h \rightarrow 0} \mathcal{E}_{V}(h)=0$.

For reference, we mention the analogous result for the Schrödinger case. If $V \in L^{5 / 2}\left(\mathbb{R}^{3}\right)$, we have for all $h>0$ and $\beta \in[0, \infty]$

$$
-C \int[V]_{+}^{5 / 2} \leq h^{3} E^{\mathrm{S}}(\beta, h, V) \leq-\frac{1}{15 \pi^{2}} \int[V]_{+}^{5 / 2}+\mathcal{E}_{V}(h)
$$

with some positive constant $C$ and with an error function depending on $V$ and $h$ and again satisfying $\lim _{h \rightarrow 0} \mathcal{E}_{V}(h)=0$. The lower bound in (6) is the classical Lieb-Thirring inequality [LT], which holds also for magnetic Schrödinger operators (see e.g., [S]) . The upper bound is achieved by setting $A=0$ and using the standard Weyl semiclassical estimate for the non-magnetic operator.

Not only does Theorem 2.1 give the almost sharp asymptotics of $h^{3} E^{\mathrm{P}}(\beta, h, V)$ in the limit of small $\kappa=\beta h$, it also essentially gives the optimal condition on which $L^{p}$-norms of $V$ are needed to bound the energy $h^{3} E^{\mathrm{P}}\left(\kappa h^{-1}, h, V\right)$ uniformly in $h$ for small $h$ and fixed $\kappa$. From the lower bound in the theorem the $L^{5 / 2}$ and $L^{4}$ norms bound the energy. Conversely, from the upper bound all $L^{p}$ norms with $p \in[5 / 2,4)$ are needed since if $V_{n} \in C_{0}^{1}$ is a sequence such that $\left\|\left[V_{n}\right]_{+}\right\|_{p} \rightarrow \infty$ as $n \rightarrow \infty$ for some $p \in[5 / 2,4)$, there is a sequence $h_{n}$ tending to zero such that $h_{n}^{3} E^{\mathrm{P}}\left(\kappa h_{n}^{-1}, h_{n}, V\right) \rightarrow-\infty$.

Remark 1. We can also ask a slightly different question: What $L^{p}$-norm condition on $V$ will ensure finiteness of the energy, but not necessarily a bound in terms of this norm? For the Schrödinger operator the answer is that $V_{+} \in L^{3 / 2}\left(\mathbb{R}^{3}\right)$ ensures finiteness of $E^{\mathrm{S}}(\beta, h, V)$ and this is essentially sharp as far as the local singularity is concerned, since for the critical case $V(x)=c|x|^{-2}$ the energy is finite if $c \leq h^{2} / 4$ and infinite otherwise. For the Pauli case we do not know a similar sharp result.

If we ask instead for finiteness of only one eigenvalue, i.e., the one-electron energy

$$
E_{0}^{\mathrm{S}, \mathrm{P}}(\beta, h, V)=\inf _{A}\left[\inf \operatorname{Spec}\left(T_{h}^{\mathrm{S}, \mathrm{P}}(A)-V\right)+\beta \int|\nabla \times A|^{2}\right]
$$

we have a sharp result for both Pauli and Schrödinger. Of course $E_{0}(\beta, h, V) \geq E(\beta, h, V)$ and hence instability for $E_{0}$ implies instability for $E$. The situation for Schrödinger is exactly the same for $E_{0}^{\mathrm{S}}$ and $E^{\mathrm{S}}$, i.e. if $V_{+} \in L^{3 / 2}\left(\mathbb{R}^{3}\right)$ then $E_{0}^{\mathrm{S}}$ is finite and for the critical case $V(x)=c|x|^{-2}$ the energy is finite if $c \leq h^{2} / 4$ and infinite otherwise.

For Pauli $E_{0}^{\mathrm{p}}$ is finite if $V_{+} \in L^{3}\left(\mathbb{R}^{3}\right)$ and for the critical case $V(x)=c|x|^{-1}$ there exists a critical value $\gamma_{\mathrm{cr}}>0$ such that $E_{0}^{\mathrm{p}}$ is finite if $c<\gamma_{\mathrm{cr}} \beta h^{2}$ and infinite if $c>\gamma_{\mathrm{cr}} \beta h^{2}$. This is essentially contained in FLL and we shall review it briefly in Appendix C. We will also discuss in the appendix that the sum of all eigenvalues $E^{\mathrm{p}}$ likewise remains bounded for the cutoff Coulomb potential $V(x)=\left[c|x|^{-1}-1\right]_{+}$if $c>0$ is small enough.

Finally, we give the result of the exact spectral asymptotics in the case of weak magnetic fields. 
Theorem 2.2 (Semiclassics for weak fields). Assume that $V \in L^{5 / 2}\left(\mathbb{R}^{3}\right) \cap L^{4}\left(\mathbb{R}^{3}\right)$ then

$$
\lim _{\substack{h \rightarrow 0 \\ \beta h \rightarrow \infty}} h^{3} E^{\mathrm{P}}(\beta, h, V)=\lim _{h \rightarrow 0} h^{3} E^{\mathrm{P}}(\infty, h, V)=-\frac{2}{15 \pi^{2}} \int[V(x)]_{+}^{5 / 2} \mathrm{~d} x .
$$

Likewise for the Schrödinger case we have for $V \in L^{5 / 2}\left(\mathbb{R}^{3}\right)$

$$
\lim _{\substack{h \rightarrow 0 \\ \beta h \rightarrow \infty}} h^{3} E^{\mathrm{S}}(\beta, h, V)=\lim _{h \rightarrow 0} h^{3} E^{\mathrm{S}}(\infty, h, V)=-\frac{1}{15 \pi^{2}} \int[V(x)]_{+}^{5 / 2} \mathrm{~d} x .
$$

Remark 2. This result is a strengthening of Theorem 1.3 from ES3, where the same conclusion was proved under the condition $\beta h^{2} \geq c>0$.

Remark 3. We conjecture that in case of the Schrödinger operator the Weyl term is the correct asymptotics uniformly in $\beta$, i.e. if $V \in L^{5 / 2}\left(\mathbb{R}^{3}\right)$, we conjecture that

$$
\lim _{h \rightarrow 0} h^{3} E^{\mathrm{S}}(\beta, h, V)=-\frac{1}{15 \pi^{2}} \int[V(x)]_{+}^{5 / 2} \mathrm{~d} x
$$

holds uniformly in $\beta \in[0, \infty)$. The upper bound (5) shows that the same statement cannot hold for the Pauli case.

Remark 4. Subleading error estimates are established first in [EFS2] and later in [Iv3].

Remark 5. The subleading error estimates in EFS2 are used in EFS3 to give the two term energy asymptotics, i.e., up to the Scott correction, in the large nuclear charge limit for atoms and molecules in a self-generated magnetic field.

\section{Stability bounds: Proof of Theorem 2.1}

The lower bound in Theorem 2.1 establishes the stability of the system, i.e., boundedness from below of the energy. As the sum of the negative eigenvalues for the Pauli operator $T_{h}^{\mathrm{P}}(A)-V$ goes to minus infinity (if $[V]_{+} \neq 0$ ) as the magnetic field increases (e.g. for a constant magnetic field [AHS]), the addition of the field energy is necessary for stability. Moreover, stability will also require sufficient decay of the potential $V$ at infinity and control of the local singularities.

The basic tool for the proof in the Pauli case is the magnetic Lieb-Thirring inequality from [LLS]:

Theorem 3.1 (Magnetic Lieb-Thirring inequality [LLS]). There exists a universal constant $C>0$ such that for the Pauli operator $T_{h}(A)-V$ with a potential $V \in L_{\text {loc }}^{1}\left(\mathbb{R}^{3}\right)$ and $V_{+} \in$ $L^{5 / 2}\left(\mathbb{R}^{3}\right) \cap L^{4}\left(\mathbb{R}^{3}\right)$ and magnetic field $B=\nabla \times A \in L^{2}\left(\mathbb{R}^{3}\right)$ we have for all $h>0$

$$
\operatorname{Tr}\left[T_{h}(A)-V\right]_{-} \geq-C h^{-3} \int[V]_{+}^{5 / 2}-C\left(h^{-2} \int|B|^{2}\right)^{3 / 4}\left(\int[V]_{+}^{4}\right)^{1 / 4} .
$$


Proof of the lower bound in Theorem 2.1. Using the magnetic Lieb-Thirring inequality (11), we have

$$
\begin{aligned}
\operatorname{Tr}\left[T_{h}(A)-V\right]_{-} & +\beta \int|\nabla \times A|^{2} \\
& \geq-C h^{-3} \int[V]_{+}^{5 / 2}-C h^{-3 / 2}\left(\int[V]_{+}^{4}\right)^{1 / 4}\left(\int B^{2}\right)^{3 / 4}+\beta \int B^{2} \\
& \geq-C h^{-3} \int[V]_{+}^{5 / 2}-C h^{-3}(\beta h)^{-3} \int[V]_{+}^{4},
\end{aligned}
$$

where we set $B=\nabla \times A$ and optimized over $\int B^{2}$.

\subsection{Upper bound in Theorem 2.1}

In order to construct a trial state that will give the upper bound in Theorem 2.1 we first use the method of [ES2] to show that there exist compactly supported magnetic fields and corresponding Pauli operators with zero-modes with arbitrarily fast decay. The original construction of zero-modes in [LY] will neither lead to compactly supported magnetic fields nor arbitrarily fast decaying modes.

Proposition 3.2. Given $m \in \mathbb{N}$. There exist a smooth magnetic field of compact support $B: \mathbb{R}^{3} \rightarrow \mathbb{R}^{3}$ with a corresponding smooth vector potential $A: \mathbb{R}^{3} \rightarrow \mathbb{R}^{3}$, a smooth and non-vanishing $\psi \in C^{\infty}\left(\mathbb{R}^{3} ; \mathbb{C}^{2}\right)$, and a constant $C>0$ such that

$$
\boldsymbol{\sigma} \cdot(-i \nabla+A) \psi=0
$$

and

$$
|\psi(x)| \leq C|x|^{-m-1} .
$$

Proof. We use the construction and notations from [ES2]. Consider the map

$$
\Phi: \mathbb{R}^{3} \ni x=\left(x_{1}, x_{2}, x_{3}\right) \mapsto 2 \frac{x_{3}+i\left(-1+|x|^{2} / 4\right)}{x_{1}+i x_{2}} \in \mathbb{C} \cup\{\infty\} .
$$

This map is $\Phi=\tau_{2} \circ \phi \circ \tau_{3}^{-1} \mid \mathbb{R}^{3}$, where $\tau_{3}: \mathbb{S}^{3} \rightarrow \mathbb{R}^{3} \cup\{\infty\}$, and $\tau_{2}: \mathbb{S}^{2} \rightarrow \mathbb{R}^{2} \cup\{\infty\}$ are stereographic projections (we have identified $\mathbb{C}$ and $\mathbb{R}^{2}$ ) and $\phi: \mathbb{S}^{3} \rightarrow \mathbb{S}^{2}$ is the Hopf map (see Lemma 34 in [ES2]). Note that

$$
1+\frac{1}{4}|\Phi(x)|^{2}=\frac{\left(1+|x|^{2} / 4\right)^{2}}{x_{1}^{2}+x_{2}^{2}} \geq|x|^{2} / 16
$$

Consider the real 2-form on $\mathbb{C}$, which by stereographic projection pulls back to (1/4 times) the volume form on $\mathbb{S}^{2}$, i.e.,

$$
\omega=\frac{1}{8}\left(1+|z|^{2} / 4\right)^{-2} i d z \wedge d \bar{z} .
$$


We have $\int_{\mathbb{C}} \omega=\pi$. For $g$ a real smooth compactly supported function $g \in C_{0}^{\infty}(\mathbb{C})$ we define the 2 -form

$$
\beta_{3}=\Phi^{*}(g \omega)
$$

on $\mathbb{R}^{3}$. Note that $\beta_{3}$ is a closed 2-form. We can therefore define a (divergence free) vector field $B$ such that $B \cdot(\mathbf{X} \times \mathbf{Y})=\beta_{3}(\mathbf{X}, \mathbf{Y})$ for all vector fields $\mathbf{X}$ and $\mathbf{Y}$. By (13), $\beta_{3}$ and hence $B$ has compact support in $\mathbb{R}^{3}$. Moreover we will assume that $(2 \pi)^{-1} \int_{\mathbb{C}} g \omega=m+\frac{1}{2}$.

Consider also the real 2-form $\beta_{2}=(g-1) \omega$ on $\mathbb{C}$. It satisfies $(2 \pi)^{-1} \int_{\mathbb{C}} \beta_{2}=m$. If we set

$$
h(z)=\pi^{-1} \int_{\mathbb{C}} \ln \left|z-z^{\prime}\right|^{2} \beta_{2}\left(z^{\prime}\right),
$$

then $\beta_{2}=d \alpha_{2}$, where $\alpha_{2}$ is the real 1-form $\alpha_{2}=2 \operatorname{Re}\left(\frac{i}{4} \partial_{\bar{z}} h(z) d \bar{z}\right)$. According to the AharonovCasher Theorem (See Theorem 37 and Appendix A in [ES2]) the magnetic Dirac-operator on $\mathbb{C}$ corresponding to the metric with volume form $\omega$ and conformal to the standard metric and one form (magnetic vector potential) $\alpha_{2}$ has a (unnormalized) zero-mode, i.e., element in the kernel, of the form $f(z)\left(\begin{array}{l}1 \\ 0\end{array}\right)$, where

$$
f(z)=\left(1+|z|^{2} / 4\right)^{1 / 2} \exp (-h(z) / 4) .
$$

In the standard metric on $\mathbb{C}$ the same would be true with a zero-mode without the prefactor $\left(1+|z|^{2} / 4\right)^{1 / 2}$, which comes from the conformal factor according to Theorem 23 in [ES2].

Note that $|f(z)| \leq C|z|^{1-m}$. In fact, there will be $m$ zero-modes, but we need only the one given above which has the fastest decay.

Let us turn to the construction of the zero-mode in $\mathbb{R}^{3}$. Let $\Omega: \mathbb{R}^{3} \rightarrow \mathbb{R}^{3}$ be the vector field corresponding to the 2 -form $\Phi^{*}(\omega)$, i.e., such that $\Omega \cdot(\mathbf{X} \times \mathbf{Y})=\omega\left(\Phi_{*}(\mathbf{X}), \Phi_{*}(\mathbf{Y})\right)$. We may choose a smooth unit vector field $\xi: \mathbb{R}^{3} \rightarrow \mathbb{C}^{2}$ such that $\boldsymbol{\sigma} \cdot \boldsymbol{\Omega} \xi=|\boldsymbol{\Omega}| \xi$. In fact, an explicit choice is

$$
\xi(x)=\left(1+|x|^{2} / 4\right)^{-1 / 2}\left(1+\frac{i}{2} \boldsymbol{\sigma} \cdot x\right)\left(\begin{array}{l}
1 \\
0
\end{array}\right) .
$$

According to Section 8 in [ES2] we can find a smooth $A$ with $\nabla \times A=B$ and such that $\boldsymbol{\sigma} \cdot(-i \nabla+A) \psi=0$, where

$$
\psi(x)=\left(1+|x|^{2}\right)^{-1} f(\Phi(x)) \xi(x) .
$$

Here again $\left(1+|x|^{2}\right)^{-1}$ is the conformal factor coming from the stereographic projection $\tau_{3}$ (see Theorem 23 in [ES2]). Thus we see that $|\psi(x)| \leq C|x|^{-m-1}$.

We can use this proposition to construct a low energy one-electron state localized in a ball.

Proposition 3.3. For all $0<\delta<1$ there is a constant $C_{\delta}>0$ such that for all $h, \beta>0$ and all balls $B_{R}$ with radius $R$ we can find a smooth magnetic field $B$ supported in $B_{R}$ such that for 
all smooth magnetic vector potentials $A$ with $\nabla \times A=B$ in $B_{R}$ we can find an $L^{2}$-normalized $\psi \in C_{0}^{\infty}\left(B_{R}, \mathbb{C}^{2}\right)$ satisfying

$$
\int|\boldsymbol{\sigma} \cdot(-i h \nabla+A) \psi|^{2}+\beta \int B^{2} \leq C_{\delta} h^{2} \beta^{1-\delta} R^{-1-\delta} .
$$

Proof. This is a simple localization and scaling argument based on the result in the previous proposition. First note that we may assume that $\beta R$ is small enough. In fact, if $\beta R>c$ for some constant $c$ then $h^{2} \beta^{1-\delta} R^{-1-\delta} \geq c^{1-\delta} h^{2} R^{-2}$ and an upper bound of the form $C h^{2} R^{-2}$ can be achieved by choosing $A=B=0$.

Without loss of generality we may assume that the ball $B_{R}$ is centered at the origin. Choose an integer $m$ such that $(2 m)^{-1} \leq \delta$. Let $\widetilde{\psi}$ be a zeromode as constructed in Proposition 3.2 , i.e,

$$
\boldsymbol{\sigma} \cdot(-i \nabla+\widetilde{A}) \widetilde{\psi}=0
$$

with decay $|\widetilde{\psi}(x)| \leq C|x|^{-m-1}$ and corresponding magnetic field $\widetilde{B}=\nabla \times \widetilde{A}$ of compact support. We may assume that $\widetilde{\psi}$ is normalized. For $\ell>0$ we define

$$
\psi_{\ell}(x)=\ell^{-3 / 2} \widetilde{\psi}(x / \ell), \quad A_{\ell}(x)=\ell^{-1} h \widetilde{A}(x / \ell)
$$

Then $B_{\ell}(x)=h \ell^{-2} \widetilde{B}(x / \ell)$ and

$$
\boldsymbol{\sigma} \cdot\left(-i h \nabla+A_{\ell}\right) \psi_{\ell}=0
$$

We can assume $\widetilde{B}$ to be supported in a ball of radius 1 centered at the origin (otherwise we rescale as just explained). Hence $B_{\ell}$ is supported in a ball of radius $\ell$.

Choose $\chi \in C_{0}^{\infty}\left(\mathbb{R}^{3}\right)$ with support in the unit ball centered at the origin and such that $0 \leq \chi(x) \leq 1$ for all $x$ and $\chi(x)=1$ if $|x|<1 / 2$. Set

$$
\psi(x)=\mathcal{N}^{-1} \chi(x / R) \psi_{\ell}(x) .
$$

where the normalization constant is $\mathcal{N}=\left(\int\left|\chi(x / R) \psi_{\ell}(x)\right|^{2} \mathrm{~d} x\right)^{1 / 2}$. Then $\psi$ is supported in $B_{R}$. Moreover, $1-C(\ell / R)^{2 m-1} \leq \mathcal{N}^{2} \leq 1$, and thus if $R>2 \ell$

$$
\begin{aligned}
\int\left|\boldsymbol{\sigma} \cdot\left(-i h \nabla+A_{\ell}\right) \psi\right|^{2} & =h^{2} \mathcal{N}^{-2} \int(\nabla(\chi(x / R)))^{2}\left|\psi_{\ell}(x)\right|^{2} \mathrm{~d} x \\
& \leq C h^{2} R^{-2}(\ell / R)^{2 m-1}
\end{aligned}
$$

Hence

$$
\begin{aligned}
\int\left|\boldsymbol{\sigma} \cdot\left(-i h \nabla+A_{\ell}\right) \psi\right|^{2}+\beta \int B_{\ell}^{2} & \leq C h^{2} R^{-2}(\ell / R)^{2 m-1}+C h^{2} \beta \ell^{-1} \\
& =C h^{2} \beta R^{-1}(\beta R)^{-\frac{1}{2 m}}
\end{aligned}
$$


with the optimal choice $\ell=C R(\beta R)^{\frac{1}{2 m}} \leq R / 2$ if $\beta R$ is small enough.

Finally, if $A$ is any smooth vector potential with $\nabla \times A=B_{\ell}$ in $B_{R}$ then we can gauge transform, i.e., find a smooth $\phi: B_{R} \rightarrow \mathbb{R}$ such that $A_{\ell}=A+\nabla \phi$ in $B_{R}$. Then

$$
\boldsymbol{\sigma} \cdot(-i h \nabla+A) e^{i h^{-1} \phi} \psi=e^{i h^{-1} \phi} \boldsymbol{\sigma} \cdot\left(-i h \nabla+A_{\ell}\right) \psi
$$

and thus the above bound holds with $\psi$ replaced by $e^{-i h^{-1} \phi} \psi$.

Proof of the upper bound in Theorem 2.1. By choosing $A=0$ we can always achieve a Weyl upper bound

$$
h^{3} E(\beta, h, V) \leq-C \int[V]_{+}^{5 / 2}+\mathcal{E}_{V}(h) .
$$

Let us now show that if $(\beta h)^{-3+2 \varepsilon} \int[V]_{+}^{4-\varepsilon}>\int[V]_{+}^{5 / 2}$ then we can achieve the bound

$$
h^{3} E(\beta, h, V) \leq-C_{\varepsilon}(\beta h)^{-3+2 \varepsilon} \int[V]_{+}^{4-\varepsilon}
$$

for $h$ small enough depending on $V$ and $\varepsilon$. Divide space into cubes of side length $\sqrt{h}$. For each cube consider the minimal value $V_{\min }$ of $V$. Then in the cube $V \leq V_{\min }+\sqrt{3 h}\|\nabla V\|_{\infty}$. If $V_{\min } \leq \sqrt{h}\|\nabla V\|_{\infty}$ we do nothing in the cube. If we denote the union of all these cubes $W Q$ (for weak cubes) we find that

$$
\int_{W Q}[V]_{+}^{4-\varepsilon} \leq C_{V} h^{2-\varepsilon / 2}
$$

for $C_{V}>0$ a constant depending only on $V$ (in particular on the support of $V$ ).

In each cube where $V_{\min } \geq \sqrt{h}\|\nabla V\|_{\infty}$ we have $V \leq(1+\sqrt{3}) V_{\min }$. Each of these cubes we fill with the maximal number of disjoint balls of radius

$$
R=\kappa_{2} h(\beta h)^{1-2 \varepsilon / 3} V_{\min }^{-1+\varepsilon / 3},
$$

where $\kappa_{2}>0$ is a constant which we will choose below depending only on $\varepsilon$. Note that

$$
R \leq \kappa_{2}^{-1+\varepsilon / 3} h^{1 / 2+\varepsilon / 6}(\beta h)^{1-2 \varepsilon / 3}\|\nabla V\|_{\infty}^{-1+\varepsilon / 3} \leq \sqrt{h} / 2
$$

if $h$ is small enough depending only on $V$ and $\varepsilon$ (recall that $\beta h$ is bounded from above in terms of $V$ ). In particular, we can then fit at least one ball in the cube.

In each of these balls we choose a magnetic field according to Proposition 3.3 with $\delta$ chosen such that $3 \delta(1+\delta)^{-1}=\varepsilon$. Let $B: \mathbb{R}^{3} \rightarrow \mathbb{R}^{3}$ be the sum of all these disjointly supported magnetic fields and let $A$ be a corresponding vector potential. Let $B^{(i)}$ and $R_{i}$ for $i=1,2, \ldots$ denote the balls (there are only finitely many) and their radii. We can according to Proposition 3.3 for each $i$ find a normalized $\psi_{i}$ supported in $B^{(i)}$ such that

$$
\begin{aligned}
\int\left|\boldsymbol{\sigma} \cdot(-i h \nabla+A) \psi_{i}\right|^{2}-\int\left|\psi_{i}\right|^{2} V+\beta \int_{B^{(i)}} B^{2} & \leq C_{\delta} h^{2} \beta^{1-\delta} R_{i}^{-1-\delta}-V_{\min , i} \\
& \leq\left(C_{\delta} \kappa_{2}^{-1-\delta}-1\right) V_{\min , i} \\
& \leq-V_{\min , i} / 2
\end{aligned}
$$


if $\kappa_{2}$ is large enough depending on $\delta$, i.e., on $\varepsilon$. Here $V_{\min , i}$ is the minimum of $V$ in the cube containing the ball $B^{(i)}$.

Since all the constructed balls are disjoint we have that $P=\sum_{i}\left|\psi_{i}\right\rangle\left\langle\psi_{i}\right|$ is an orthogonal projection and hence

$$
\begin{aligned}
E(\beta, h, V) & \leq \operatorname{Tr}\left[\left((\boldsymbol{\sigma} \cdot(-i h \nabla+A))^{2}-V\right) P\right]+\beta \int B^{2} \\
& \leq \sum_{i}-V_{\min , i} / 2=\sum_{i}-\frac{1}{2} \kappa_{2}^{-3} h^{-3}(\beta h)^{-3+2 \varepsilon} V_{\min , i}^{4-\varepsilon} R_{i}^{3} \\
& \leq-2 C_{\varepsilon} h^{-3}(\beta h)^{-3+2 \varepsilon} \int_{\mathbb{R}^{3} \backslash W Q} V^{4-\varepsilon},
\end{aligned}
$$

for some constant $C_{\varepsilon}>0$ depending only on $\varepsilon$. We have here used that in each of the cubes the balls take up a certain fraction bounded below of the volume and that $V \leq(1+\sqrt{3}) V_{\min }$. Let us emphasize that $\kappa_{2}$ was chosen depending only on $\varepsilon$. This allows us to ensure that (16) is satisfied if $h$ is small enough depending on $V$ and $\varepsilon$. Finally, from (15) we can choose $h$ so small depending on $V$ and $\varepsilon$ that

$$
\int_{W Q}[V]_{+}^{4-\varepsilon} \leq \frac{1}{2} \int_{\mathbb{R}^{3}}[V]_{+}^{4-\varepsilon} .
$$

This proves the claim (14).

\section{Semiclassics for weak fields: Proof of Theorem 2.2}

Proof of Theorem 2.9. We discuss only the Pauli case, the Schrödinger case is similar but much easier and is left to the reader. In the remaining part of the proof we will omit the superscript $\mathrm{P}$.

For the upper bound on $h^{3} E(\beta, h, V)$ we choose $A \equiv 0$ in the definition (11) and then we have $E(\beta, h, V) \leq E(\infty, h, V)$ and the second equality in (8) is just the usual non-magnetic semiclassical asymptotics.

For the lower bound, we first remark $V \geq 0$ can be assumed and that it is sufficient to prove the result for $V \in C_{0}^{\infty}\left(\mathbb{R}^{3}\right)$ by a standard approximation argument. The error between $V \in L^{5 / 2} \cap L^{4}$ and its $C_{0}^{\infty}$-approximation $\widetilde{V}$ can be made arbitrarily small in the $\|\cdot\|_{5 / 2}+\|\cdot\|_{4}$ norm. Thus the replacement of $V$ with $\widetilde{V}$ can be controlled by using the magnetic LiebThirring inequality (11) and borrowing a small part of the kinetic energy and the magnetic energy. For more details, see Section 5.4 of [ES3] (with the only modification that instead of (5.58) of [ES3] use (12)).

Secondly, as discussed in Appendix $₫$ we may in (1) replace $\int_{\mathbb{R}^{3}}|\nabla \times A|^{2}$ by $\int_{\mathbb{R}^{3}}|\nabla \otimes A|^{2}$, where the last integrand contains all derivatives. 
Thirdly, we may replace $E(\beta, h, V)$ with a localized version of the total energy. Let $0 \leq$ $\phi^{*}(x) \leq 1$ be a smooth function with $\operatorname{supp} \phi^{*} \subset B(1)$ and $\phi^{*} \equiv 1$ on $B(1 / 2)$, where $B(r)$ denotes the ball of radius $r$ centered at the origin. Denote by $\phi_{r}(x)=\phi^{*}(x / r)$. Using a partition of unity, $\phi_{r}^{2}+\eta_{r}^{2} \equiv 1$, and the IMS localization, we have

$$
\operatorname{Tr}\left[T_{h}(A)-V\right]_{-} \geq \operatorname{Tr}\left[\phi_{r}\left(T_{h}(A)-V-h^{2} I_{r}\right) \phi_{r}\right]_{-}+\operatorname{Tr}\left[\eta_{r}\left(T_{h}(A)-V-h^{2} I_{r}\right) \eta_{r}\right]_{-},
$$

where $I_{r}:=\left(\nabla \phi_{r}\right)^{2}+\left(\nabla \eta_{r}\right)^{2}$ is supported in the shell $\{r / 2 \leq|x| \leq r\}$. The second term is bounded by the magnetic Lieb-Thirring inequality similarly to (12). More precisely, for any $\varepsilon>0$ there is a sufficiently large $r=r_{\varepsilon} \geq 1$ such that

$$
\begin{aligned}
\operatorname{Tr}\left[\eta_{r}\left(T_{h}(A)-V-h^{2} I_{r}\right) \eta_{r}\right]_{-} & \geq \operatorname{Tr}\left(T_{h}(A)-\widehat{V}\right)_{-} \\
& \geq-C h^{-3} \int_{|x| \geq r} \widehat{V}^{5 / 2}-C h^{-3}(\beta h)^{-3}\left(\int_{|x| \geq r} \widehat{V}^{4}\right)-\frac{\beta}{2} \int|\nabla \times A|^{2} \\
& =-\varepsilon h^{-3}-\frac{\beta}{2} \int|\nabla \times A|^{2}
\end{aligned}
$$

holds if $h \leq h_{\varepsilon}$, where $\widehat{V}(x):=V(x)+h^{2} I_{r}$. Here we used the integrability conditions on $V$ and that $\beta h$ is bounded from below.

It is therefore sufficient to give a lower bound on the first term in (17), more precisely, we have

$$
E(\beta, h, V) \geq-\varepsilon h^{-3}+\inf _{A}\left[\operatorname{Tr}\left[\phi\left(T_{h}(A)-W\right) \phi\right]_{-}+\frac{\beta}{2} \int_{\mathbb{R}^{3}}|\nabla \otimes A|^{2}\right],
$$

where we set $W:=V+h^{2} I_{r}$ and $\phi=\phi_{r}$ for brevity.

To estimate the right hand side of (19), we will follow the argument of Section 5 of [ES3]. We choose a length $L$ with $h \leq L \leq h^{1 / 2}$. Let $\Omega_{L}:=B(r+L)$ be the $L$-neighborhood of $\Omega:=B(r)$. Let $Q_{k}:=\left\{y \in \mathbb{R}^{3}:\|y-k\|_{\infty}<L / 2\right\}$ with $k \in(L \mathbb{Z})^{3} \cap \Omega_{L}$ denote a nonoverlapping covering of $B(r)$ with boxes of size $L$. In this section the index $k$ will always run over the set $(L \mathbb{Z})^{3} \cap \Omega_{L}$. Let $\xi_{k}$ be a partition of unity, $\sum_{k} \xi_{k}^{2} \equiv 1$, subordinated to the collection of boxes $Q_{k}$, such that

$$
\operatorname{supp} \xi_{k} \subset(2 Q)_{k}, \quad\left|\nabla \xi_{k}\right| \leq C L^{-1}
$$

where $(2 Q)_{k}$ denotes the cube of side-length $2 L$ with center $k$. Let $\widetilde{\xi}_{k}$ be a cutoff function such that $\widetilde{\xi}_{k} \equiv 1$ on $(2 Q)_{k}$ (i.e. on the support of $\xi_{k}$ ), supp $\widetilde{\xi}_{k} \subset \widetilde{Q}_{k}:=(3 Q)_{k}$ and $\left|\nabla \widetilde{\xi}_{k}\right| \leq C L^{-1}$.

Let $\langle A\rangle_{k}=\left|\widetilde{Q}_{k}\right|^{-1} \int_{\widetilde{Q}_{k}} A, A_{k}:=\left(A-\langle A\rangle_{k}\right) \widetilde{\xi}_{k}$ and $B_{k}:=\nabla \times A_{k}$, then by Poincaré inequality we have

$$
\begin{aligned}
\int_{\mathbb{R}^{3}} B_{k}^{2} \leq \int_{\widetilde{Q}_{k}}\left|\nabla \otimes A_{k}\right|^{2} & \leq C \int_{\widetilde{Q}_{k}}|\nabla \otimes A|^{2}+C L^{-2} \int_{\widetilde{Q}_{k}}\left|A-\langle A\rangle_{k}\right|^{2} \\
& \leq C \int_{\widetilde{Q}_{k}}|\nabla \otimes A|^{2}
\end{aligned}
$$


From the IMS localization with a phase function $\psi_{k}$ satisfying $h \nabla \psi_{k}=\left\langle A_{k}\right\rangle$ we have

$$
\begin{aligned}
\operatorname{Tr}\left[\phi\left(T_{h}(A)-W\right) \phi\right]_{-}+\frac{\beta}{2} \int_{\mathbb{R}^{3}} B^{2} & =\inf _{\gamma} \operatorname{Tr}\left(\gamma \phi\left[T_{h}(A)-W\right] \phi\right)+\beta \int_{\mathbb{R}^{3}}|\nabla \otimes A|^{2} \\
& \geq \inf _{\gamma} \sum_{k \in(L \mathbb{Z})^{3} \cap \Omega_{L}} \mathcal{E}_{k}(\gamma)
\end{aligned}
$$

with

$$
\mathcal{E}_{k}(\gamma):=\operatorname{Tr}\left[\gamma \xi_{k} e^{-i \psi_{k}} \phi\left[T_{h}\left(A-\langle A\rangle_{k}\right)-W-C h^{2} L^{-2}\right] \phi e^{i \psi_{k}} \xi_{k}\right]+c_{0} \beta \int_{\widetilde{Q}_{k}}|\nabla \otimes A|^{2}
$$

with some universal constant $c_{0}$ and after reallocating the localization error. In (21) the infimum is taken over all density matrices $0 \leq \gamma \leq 1$. We also reallocated the second integral to account for the finite overlap of the cubes $\widetilde{Q}_{k}$. We introduce the notation

$$
\mathcal{F}_{k}:=c_{0} \beta \int_{\widetilde{Q}_{k}}|\nabla \otimes A|^{2} .
$$

Let $[H]_{Q}$ denote the operator $H$ with Dirichlet boundary conditions on the box $Q$. For each fixed box $\widetilde{Q}_{k}$ we apply the magnetic Lieb-Thirring inequality [LLS] together with (20) to obtain that for any density matrix $\gamma$

$$
\begin{aligned}
\mathcal{E}_{k}(\gamma) & \geq \operatorname{Tr}\left[\left[T_{h}\left(A_{k}\right)-W-C h^{2} L^{-2}\right]_{\widetilde{Q}_{k}}\right]_{-}+\mathcal{F}_{k} \\
& \geq-C h^{-3} \int_{\widetilde{Q}_{k}}\left[W+C h^{2} L^{-2}\right]^{5 / 2}-C\left(\int_{\widetilde{Q}_{k}}\left[W+C h^{2} L^{-2}\right]^{4}\right)^{1 / 4}\left(h^{-2} \int_{\widetilde{Q}_{k}} B_{k}^{2}\right)^{3 / 4}+\mathcal{F}_{k} \\
& \geq-C h^{-3} L^{3}-C h^{-6} L^{3} \beta^{-3}-\frac{c_{0}}{2} \beta \int_{\widetilde{Q}_{k}}|\nabla \otimes A|^{2}+\mathcal{F}_{k} \\
& \geq-C h^{-3} L^{3}+\frac{1}{2} \mathcal{F}_{k}
\end{aligned}
$$

using $h \leq L$ and $\beta h \rightarrow \infty$. The constants $C$ depend on $\|W\|_{\infty}$.

Let $S \subset(L \mathbb{Z})^{3} \cap \Omega_{L}$ denote the set of those $k$ indices such that

$$
\mathcal{F}_{k} \leq C_{1} h^{-3} L^{3}
$$

holds with some large constant $C_{1}$. In particular, by choosing $C_{1}$ sufficiently large, we have

$$
\mathcal{E}_{k}(\gamma) \geq 0, \quad \text { for all } k \notin S \text { and for any } \gamma \text {. }
$$

We use the Schwarz inequality in the form

$$
T_{h}\left(A-\langle A\rangle_{k}\right) \geq-\left(1-\varepsilon_{k}\right) h^{2} \Delta-C \varepsilon_{k}^{-1}\left(A-\langle A\rangle_{k}\right)^{2},
$$


with some $0<\varepsilon_{k}<\frac{1}{3}$, then

$$
\begin{aligned}
\mathcal{E}_{k}(\gamma) \geq & \operatorname{Tr}\left[\phi \xi_{k}\left[-\left(1-2 \varepsilon_{k}\right) h^{2} \Delta-W-C h^{2} L^{-2}\right] \xi_{k} \phi\right]_{-} \\
& +\operatorname{Tr}\left[\mathbf{1}_{\widetilde{Q}_{k}}\left[-\varepsilon_{k} h^{2} \Delta-C \varepsilon_{k}^{-1}\left(A-\langle A\rangle_{k}\right)^{2}\right] \mathbf{1}_{\widetilde{Q}_{k}}\right]_{-}+\mathcal{F}_{k} .
\end{aligned}
$$

We will show at the end of the section that

$$
\begin{aligned}
\operatorname{Tr}\left[\phi \xi_{k}[-(1\right. & \left.\left.\left.-2 \varepsilon_{k}\right) h^{2} \Delta-W-C h^{2} L^{-2}\right] \xi_{k} \phi\right]_{-} \\
& \geq \operatorname{Tr}\left[\phi \xi_{k}\left(-h^{2} \Delta-W\right) \xi_{k} \phi\right]_{-}-C h^{-3}\left(\varepsilon_{k}+h^{2} L^{-2}\right)\left|\widetilde{Q}_{k}\right| .
\end{aligned}
$$

Using (23) and (25),

$$
\begin{aligned}
\inf _{\gamma} \sum_{k} \mathcal{E}_{k}(\gamma) & \geq \inf _{\gamma} \sum_{k \in S} \mathcal{E}_{k}(\gamma) \\
& \geq \sum_{k} \operatorname{Tr}\left[\phi \xi_{k}\left[-h^{2} \Delta-W\right] \xi_{k} \phi\right]_{-}+\sum_{k \in S} \mathcal{D}_{k} \\
& \geq \sum_{k} \inf _{\gamma_{k}} \operatorname{Tr}\left[\xi_{k} \gamma_{k} \xi_{k} \phi\left[-h^{2} \Delta-W\right] \phi\right]+\sum_{k \in S} \mathcal{D}_{k} \\
& \geq \operatorname{Tr}\left[\phi\left(-h^{2} \Delta-W\right) \phi\right]_{-}+\sum_{k \in S} \mathcal{D}_{k}
\end{aligned}
$$

with

$$
\mathcal{D}_{k}:=\operatorname{Tr}\left[\left[-\varepsilon_{k} h^{2} \Delta-C \varepsilon_{k}^{-1}\left(A-\langle A\rangle_{k}\right)^{2}\right]_{\widetilde{Q}_{k}}\right]_{-}-C h^{-3}\left|\widetilde{Q}_{k}\right|\left(\varepsilon_{k}+h^{2} L^{-2}\right)+\mathcal{F}_{k} .
$$

In the last step in (26) we used that for any collection of density matrices $\gamma_{k}$, the density matrix $\sum_{k} \xi_{k} \gamma_{k} \xi_{k}$ is admissible in the variational principle

$$
\operatorname{Tr}\left[\phi\left(-h^{2} \Delta-W\right) \phi\right]_{-}=\inf \left\{\operatorname{Tr} \gamma\left[-h^{2} \Delta-W\right]: 0 \leq \gamma \leq 1,\right\} .
$$

We estimate $\mathcal{D}_{k}$ for $k \in S$ as follows

$$
\begin{aligned}
\mathcal{D}_{k} & \geq-C \varepsilon_{k}^{-4} h^{-3} \int_{\widetilde{Q}_{k}}\left(A-\langle A\rangle_{k}\right)^{5}-C h^{-3}\left|\widetilde{Q}_{k}\right|\left(\varepsilon_{k}+h^{2} L^{-2}\right)+\mathcal{F}_{k} \\
& \geq \mathcal{F}_{k}-C \varepsilon_{k}^{-4} h^{-3} \beta^{-5 / 2} L^{1 / 2} \mathcal{F}_{k}^{5 / 2}-C h^{-3}\left|\widetilde{Q}_{k}\right|\left(\varepsilon_{k}+h^{2} L^{-2}\right) .
\end{aligned}
$$

In the first step we used Lieb-Thirring inequality, in the second step Hölder and Sobolev inequalities in the form

$$
\int_{\widetilde{Q}_{k}}\left(A-\langle A\rangle_{k}\right)^{5} \leq C L^{1 / 2}\left(\int_{\widetilde{Q}_{k}}|\nabla \otimes A|^{2}\right)^{5 / 2} .
$$


We choose

$$
\varepsilon_{k}=\beta^{-1 / 2} L^{-1 / 2} \mathcal{F}_{k}^{1 / 2}
$$

and using the a priori bound (22), we see that

$$
\varepsilon_{k} \leq C h^{-1} L(\beta h)^{-1 / 2} .
$$

Thus, choosing

$$
L=h(\beta h)^{1 / 10}
$$

we get $\varepsilon_{k} \leq C(\beta h)^{-2 / 5} \leq 1 / 3$ as $\beta h \rightarrow \infty$. With this choice of $\varepsilon_{k}$ and $L$ we have from (29)

$$
\mathcal{D}_{k} \geq \mathcal{F}_{k}-C(h \beta)^{-1 / 4} \mathcal{F}_{k}^{1 / 2}-C h^{-3} L^{3}(\beta h)^{-1 / 5} \geq-C h^{-3} L^{3}(\beta h)^{-1 / 5} .
$$

Summing up (31) for all $k$ and using that

$$
\sum_{k \in(L \mathbb{Z})^{3} \cap \Omega_{L}} L^{3} \leq C r^{3},
$$

we obtain from (26) and (31)

$$
\begin{aligned}
\inf _{\gamma} \sum_{k} \mathcal{E}_{k}(\gamma) & \geq \operatorname{Tr}\left[\phi\left(-h^{2} \Delta-W\right) \phi\right]_{-}-C h^{-3}(\beta h)^{-1 / 5} r^{3} \\
& \geq-\frac{2}{15 \pi^{2}}(1+o(1)) \int W^{5 / 2}-C h^{-3}(\beta h)^{-1 / 5} r^{3}
\end{aligned}
$$

using the standard semiclassical asymptotics for $\operatorname{Tr}\left[\phi\left(-h^{2} \Delta-W\right) \phi\right]_{-} \geq \operatorname{Tr}\left(-h^{2} \Delta-W\right)_{-}$. Together with (21) this proves the required lower bound for the second term in (19). The difference between $\int W^{5 / 2}$ and $\int V^{5 / 2}$ is negligible as $h \rightarrow 0$. Letting first $h \rightarrow 0$ together with $\beta h \rightarrow \infty$ and then letting $\varepsilon \rightarrow 0$ we obtain the lower bound in (8).

Finally, we prove (25). Let $\gamma$ be a trial density matrix for the left hand side of (25). We can assume that

$$
0 \geq \operatorname{Tr}\left[\gamma \phi \xi_{k}\left[-\left(1-2 \varepsilon_{k}\right) h^{2} \Delta-W-C h^{2} L^{-2}\right] \xi_{k} \phi\right] .
$$

Recalling that $\varepsilon_{k} \leq \frac{1}{3}$, we have

$$
\begin{aligned}
0 & \geq \operatorname{Tr}\left[\gamma \phi \xi_{k}\left[-\frac{1}{6} h^{2} \Delta+1\right] \xi_{k} \phi\right]+\operatorname{Tr}\left[\gamma \phi \xi_{k}\left[-\frac{1}{6} h^{2} \Delta-W-C h^{2} L^{-2}-1\right] \xi_{k} \phi\right] \\
& \geq \operatorname{Tr}\left[\gamma \phi \xi_{k}\left[-\frac{1}{6} h^{2} \Delta+1\right] \xi_{k} \phi\right]-C h^{-3} \int_{\widetilde{Q}_{k}}\left[W+1+C h^{2} L^{-2}\right]^{5 / 2},
\end{aligned}
$$

where we used Lieb-Thirring inequality. Thus, using $h \leq L$, we have

$$
\operatorname{Tr}\left[\gamma \phi \xi_{k}\left[-\frac{1}{6} h^{2} \Delta+1\right] \xi_{k} \phi\right] \leq C h^{-3}\left|\widetilde{Q}_{k}\right|
$$


with a constant depending on $W$. Therefore

$$
\begin{aligned}
\operatorname{Tr}\left[\gamma \phi \xi_{k}[-(1\right. & \left.\left.\left.-2 \varepsilon_{k}\right) h^{2} \Delta-W-C h^{2} L^{-2}\right] \xi_{k} \phi\right] \\
& \geq \operatorname{Tr}\left[\gamma \phi \xi_{k}\left(-h^{2} \Delta-W\right) \xi_{k} \phi\right]-C h^{-3}\left(\varepsilon_{k}+h^{2} L^{-2}\right)\left|\widetilde{Q}_{k}\right| .
\end{aligned}
$$

Now (25) follows by the variational principle.

\section{A Equivalent forms of the energy}

We will consider the equivalence of the total energy where we have different restrictions on the vector potentials. We allow the energy to possibly have an extra localization. So we end up considering

$$
E_{\times}(A)=\operatorname{Tr}\left[\psi\left(T_{h}(A)-V\right) \psi\right]_{-}+\beta \int_{\mathbb{R}^{3}}|\nabla \times A|^{2},
$$

where $\psi \in C^{\infty}\left(\mathbb{R}^{3}\right)$ satisfies $0 \leq \psi \leq 1$. Similarly, we define

$$
E_{\otimes}(A)=\operatorname{Tr}\left[\psi\left(T_{h}(A)-V\right) \psi\right]_{-}+\beta \int_{\mathbb{R}^{3}}|\nabla \otimes A|^{2},
$$

where $|\nabla \otimes A|^{2}=\sum_{i, j=1}^{3}\left|\partial_{i} A_{j}\right|^{2}$. Some natural domains of definition are given below:

$$
\begin{aligned}
& \mathcal{D}_{1}=\left\{A \in L^{6}\left(\mathbb{R}^{3}, \mathbb{R}^{3}\right): \int|\nabla \times A|^{2}<\infty\right\} \\
& \mathcal{D}_{2}=C_{0}^{\infty}\left(\mathbb{R}^{3}, \mathbb{R}^{3}\right) \\
& \mathcal{D}_{3}=H^{1}\left(\mathbb{R}^{3}, \mathbb{R}^{3}\right) \\
& \mathcal{D}_{4}=\left\{A \in \mathcal{D}_{1}: \nabla \cdot A=0\right\}
\end{aligned}
$$

In the case of $E_{\otimes}$, the expression $\int|\nabla \times A|^{2}$ in $\mathcal{D}_{1}$ should be replaced by $\int|\nabla \otimes A|^{2}$.

We will only assume that $V \in L_{\text {loc }}^{1}\left(\mathbb{R}^{3}\right)$. The trace in the above expressions should then be interpreted as

$$
\inf \sum_{j=1}^{N}\left\langle\phi_{j} \mid \psi\left(T_{h}(A)-V\right) \psi \phi_{j}\right\rangle
$$

where $\left\{\phi_{j}\right\}_{j=1}^{N}$ runs over all orthonormal subsets of $C_{0}^{\infty}\left(\mathbb{R}^{3}\right)$. If this infimum is different from $-\infty$, it implies in particular that the quadratic form of $\psi\left(T_{h}(A)-V\right) \psi$ defined on $C_{0}^{\infty}$ is semibounded from below. In that case (37) will be equal to the trace of the negative part of the Friedrichs extension of this quadratic form, thereby justifying the notation. 
Proposition A.1. We have for all $i, j \in\{1,2,3,4\}$,

$$
\inf _{A \in \mathcal{D}_{i}} E_{\times}(A)=\inf _{A \in \mathcal{D}_{j}} E_{\otimes}(A)
$$

Notice though that we do not prove that one can both impose compact support and zero divergence at the same time.

Proof. Consider first the $E_{\times}$. Clearly, $\mathcal{D}_{2} \subset \mathcal{D}_{3} \subset \mathcal{D}_{1}$ (using the Sobolev inequality to get the last inclusion) which implies corresponding inequalities for the energies. We will now prove that $\inf _{A \in \mathcal{D}_{1}} E_{\times}(A) \geq \inf _{A \in \mathcal{D}_{2}} E_{\times}(A)$. But for any $A \in \mathcal{D}_{1}$ and any finite collection $\left\{\phi_{j}\right\} \subset C_{0}^{\infty}\left(\mathbb{R}^{3}\right)$ we can get arbitrarily close to

$$
\sum_{j=1}^{N}\left\langle\phi_{j} \mid \psi\left(T_{h}(A)-V\right) \psi \phi_{j}\right\rangle+\beta \int|\nabla \times A|^{2}
$$

by simultaneously approximating $A$ in $L^{6}$-norm and $\nabla \times A$ in $L^{2}$, by a $C_{0}^{\infty}$ vector field. Therefore

$$
\inf _{A \in \mathcal{D}_{1}} E_{\times}(A)=\inf _{A \in \mathcal{D}_{2}} E_{\times}(A)=\inf _{A \in \mathcal{D}_{3}} E_{\times}(A) .
$$

Clearly $\inf _{A \in \mathcal{D}_{1}} E_{\times}(A) \leq \inf _{A \in \mathcal{D}_{4}} E_{\times}(A)$. We will prove that $\inf _{A \in \mathcal{D}_{2}} E_{\times}(A) \geq \inf _{A \in \mathcal{D}_{4}} E_{\times}(A)$, thereby establishing the equality for all four energies $E_{\times}$. Let $A \in C_{0}^{\infty}\left(\mathbb{R}^{3}, \mathbb{R}^{3}\right)$ and $\left\{\phi_{j}\right\}_{j=1}^{N} \subset$

$C_{0}^{\infty}\left(\mathbb{R}^{3}\right)$. Then $B=\nabla \times A \in L^{2}\left(\mathbb{R}^{3}\right)$ and therefore there exists $A^{\prime} \in \mathcal{D}_{4}$ with $\nabla \times A^{\prime}=B$ (see [FLL]). It follows that there exists $\eta$ with $A-A^{\prime}=\nabla \eta$ and therefore (since $\Delta \eta=\nabla \cdot A$ ), $\eta \in C^{\infty}\left(\mathbb{R}^{3}\right)$. But then

$$
\sum_{j=1}^{N}\left\langle\phi_{j} \mid \psi\left(T_{h}(A)-V\right) \psi \phi_{j}\right\rangle=\sum_{j=1}^{N}\left\langle e^{i \eta} \phi_{j} \mid \psi\left(T_{h}\left(A^{\prime}\right)-V\right) \psi\left(e^{i \eta} \phi_{j}\right)\right\rangle
$$

which establishes the desired inequality. Since $\int|\nabla \otimes A|^{2}=\int|\nabla \times A|^{2}+\int|\nabla \cdot A|^{2}$, the same arguments give the identities for the $E_{\otimes}$ versions of the energies.

Finally we prove that $\inf _{A \in \mathcal{D}_{4}} E_{\times}(A)=\inf _{A \in \mathcal{D}_{4}} E_{\otimes}(A)$. But this is obvious since the field energies are identical when $\nabla \cdot A=0$.

\section{B Self-generated magnetic fields lower the energy}

In this appendix we will show that self-generated magnetic fields may indeed decrease the energy, i.e., inequality (3).

Proof of (3). For the Pauli operator we already remarked this fact as a consequence of Theorem 2.1. Alternatively, it also follows from the instability in (41) in Appendix C 
below, since the non-magnetic Hydrogen atom is stable. For the Schrödinger operator this statement was essentially proved in [ELV] (see also [FLW]) by considering the perturbative regime as a small magnetic field is turned on. A simple first order perturbation argument shows that the lowest eigenvalue increases quadratically in $B$. In a spherical geometry the higher non-magnetic eigenvalues are degenerate and some of them carry non-trivial current. These eigenvalues will split linearly when a small magnetic field is turned on. To see this explicitly we can consider a spherically symmetric harmonic oscillator in a constant magnetic field, i.e., $V(x)=|x|^{2}$ and $A(x, y, z)=(B y / 2,-B x / 2,0)$ with $B>0$ constant. The eigenvalues of the operator $(-i \nabla-A)^{2}+|x|^{2}$ are (see $\left.[\mathrm{Fo}]\right)$

$$
e\left(n_{1}, n_{2}, n_{3}\right)=\left(n_{1}+n_{2}+1\right) \sqrt{1+B^{2}}+\left(n_{3}+1 / 2\right)+\left(n_{1}-n_{2}\right) B
$$

with $n_{1}, n_{2}, n_{3} \in \mathbb{N}$. Thus as an explicit example

$$
\operatorname{Tr}\left((-i \nabla-A)^{2}+|x|^{2}-5 / 2\right)_{-}=3 \sqrt{1+B^{2}}-4-B
$$

which of course explicitly decreases as a small $B$ is increased from zero. It is now clear that we can find $\widetilde{A} \in C_{0}^{\infty}\left(\mathbb{R}^{3} ; \mathbb{R}^{3}\right)$ which approximates $A$ such that

$$
\operatorname{Tr}\left((-i \nabla-\widetilde{A})^{2}+|x|^{2}-5 / 2\right)_{-}<\operatorname{Tr}\left(-\Delta+|x|^{2}-5 / 2\right)_{-}=-1
$$

and hence for $\beta>0$ sufficiently small

$$
\operatorname{Tr}\left((-i \nabla-\widetilde{A})^{2}+|x|^{2}-5 / 2\right)_{-}+\beta \int|\nabla \times \widetilde{A}|^{2}<\operatorname{Tr}\left[-\Delta+|x|^{2}-5 / 2\right]_{-} .
$$

\section{Stability conditions}

Using the argument in FLL] it is easy to show the following stability result on the one-electron energy.

Proposition C.1. Let $V \in L_{l o c}^{1}\left(\mathbb{R}^{3}\right)$ with $V_{+} \in L^{3}\left(\mathbb{R}^{3}\right) \cap L^{3 / 2}\left(\mathbb{R}^{3}\right)$. Then for all $\psi \in H^{1}\left(\mathbb{R}^{3}\right)$ with $\|\psi\|_{L^{2}}=1$ we have

$$
\left\langle\psi,\left(T_{h}^{\mathrm{P}}(A)-V\right) \psi\right\rangle+\beta \int B^{2} \geq 0
$$

if $\beta^{-1} h^{-2}\left\|V_{+}\right\|_{3}$ and $h^{-2}\left\|V_{+}\right\|_{3 / 2}$ are (universally) small enough.

Proof. Let $C_{S}>0$ be the Sobolev constant, i.e., $\int|\nabla \psi|^{2} \geq C_{S}\|\psi\|_{6}^{2}$. Since

$$
T_{h}^{\mathrm{P}}(A)=(-i h \nabla+A)^{2}+h \boldsymbol{\sigma} \cdot B
$$


we estimate for all $0<\varepsilon \leq 1$

$$
\begin{aligned}
\left\langle\psi, T_{h}^{\mathrm{P}}(A) \psi\right\rangle+\beta \int B^{2} & \geq C_{S} h^{2} \varepsilon\|\psi\|_{6}^{2}-h \varepsilon \int|B \| \psi|^{2}+\beta \int B^{2} \\
& \geq C_{S} h^{2} \varepsilon\|\psi\|_{6}^{2}-(4 \beta)^{-1} \varepsilon^{2} h^{2} \int|\psi|^{4} \\
& \geq C_{S} h^{2} \varepsilon\|\psi\|_{6}^{2}-(4 \beta)^{-1} \varepsilon^{2} h^{2}\|\psi\|_{6}^{3}\|\psi\|_{2} .
\end{aligned}
$$

We will also use that for $p \geq 3 / 2$ we have the Hölder inequality

$$
\int V|\psi|^{2} \leq\left\|V_{+}\right\|_{p}\|\psi\|_{6}^{3 / p}\|\psi\|_{2}^{2-3 / p}
$$

We consider two cases.

Case 1: $\|\psi\|_{6} \leq 2 C_{S} \beta$. We set $\varepsilon=1$ and $p=3 / 2$ above and find since $\|\psi\|_{2}=1$

$$
\left\langle\psi,\left(T_{h}^{\mathrm{P}}(A)-V\right) \psi\right\rangle+\beta \int B^{2} \geq \frac{1}{2} C_{s} h^{2}\|\psi\|_{6}^{2}-\left\|V_{+}\right\|_{3 / 2}\|\psi\|_{6}^{2}
$$

from which it follows that the energy is non-negative if $\left\|V_{+}\right\|_{3 / 2} \leq C_{S} h^{2} / 2$.

Case 2: $\|\psi\|_{6} \geq 2 C_{S} \beta$. Let $\varepsilon=2 C_{S} \beta\|\psi\|_{6}^{-1} \leq 1$ and $p=3$. Then

$$
\left\langle\psi,\left(T_{h}^{\mathrm{P}}(A)-V\right) \psi\right\rangle+\beta \int B^{2} \geq C_{S}^{2} h^{2} \beta\|\psi\|_{6}-\left\|V_{+}\right\|_{3}\|\psi\|_{6} .
$$

Hence the energy is non-negative if $\left\|V_{+}\right\|_{3} \leq C_{S}^{2} h^{2} \beta$.

It follows immediately from this proposition that the one-electron energy $E_{0}^{\mathrm{P}}(\beta, h, V)$ is finite if $V_{+} \in L^{3}\left(\mathbb{R}^{3}\right)$. In fact, all we have to argue is that $\beta h^{-2}\left\|[V-e]_{+}\right\|_{3}$ and $h^{-2}\left\|[V-e]_{+}\right\|_{3 / 2}$ can be made small enough by choosing $e>0$ large enough. In this way $-e$ can be made a lower bound on $E_{0}^{\mathrm{P}}$. Since $V_{+} \in L^{3}$ we can of course make $\left\|[V-e]_{+}\right\|_{3}$ arbitrarily small. Using that $[V-e]_{+}^{3 / 2} \leq\left(2 e^{-1}\right)^{3 / 2}[V-e / 2]_{+}^{3}$ we can do the same with $\left\|[V-e]_{+}\right\|_{3 / 2}$.

This stability criterion is essentially sharp. In fact, applying the method of proof as in the proposition above and the construction of zero-modes in [LY] it was proved in [FLL] that for the Coulomb potential $V(x)=c|x|^{-1}$ there is a critical value $\gamma_{\text {cr }}$ such that the one-electron energy satisfies

$$
E_{0}^{\mathrm{P}}(\beta, h, V)>-\infty \quad \text { if } \gamma_{\mathrm{cr}} \beta h^{2}>c
$$

and

$$
E_{0}^{\mathrm{P}}(\beta, h, V)=-\infty \quad \text { if } \gamma_{\mathrm{cr}} \beta h^{2}<c .
$$

Since $E^{\mathrm{P}} \leq E_{0}^{\mathrm{P}}$ it is clear that (40) implies that even for the cutoff Coulomb potential $V=$ $\left[c|x|^{-1}-1\right]_{+}$we have

$$
E^{\mathrm{P}}(\beta, h, V)=-\infty \quad \text { if } \gamma_{\mathrm{cr}} \beta h^{2}<c .
$$


However there is also a value $\gamma_{\mathrm{cr}}^{\prime}>0$ such that

$$
E^{\mathrm{P}}(\beta, h, V)>-\infty \quad \text { if } \gamma_{\mathrm{cr}}^{\prime} \beta h^{2}>c
$$

This stability statement follows, e.g., by localizing in an appropriate ball and then follow the proof of Lemma 2.1 [ES3] for the inner regime (with the choice of $Z=h^{-2}, \delta=Z^{1 / 3}$, $D=R Z^{1 / 3}$ and $\left.16 \pi \alpha^{2}=\beta^{-1}\right)$. In the outer regime the operator has a compactly supported bounded potential (that includes the localization error) so its energy is controlled by the magnetic Lieb-Thirring inequality as in (12).

\section{References}

[AHS] J. Avron, I. Herbst and B. Simon: Schrödinger operators with magnetic fields. I. General interactions. Duke Math. J. 45 (1978), 847-883.

[ELV] L. Erdös, M. Loss and V. Vougalter, Diamagnetic behavior of sums of Dirichlet eigenvalues. Ann. Inst. Fourier (Grenoble), Vol 50, no. 3. 891-907 (2000).

[ES1] L. Erdős and J. P. Solovej: Semiclassical eigenvalue estimates for the Pauli operator with strong non-homogeneous magnetic fields. II. Leading order asymptotic estimates. Commun. Math. Phys. 188, 599-656 (1997)

[ES2] L. Erdös, J. P. Solovej, The kernel of Dirac operators on $S^{3}$ and $\mathbf{R}^{3}$. Rev. Math. Phys. 13 No. 10, 1247-1280 (2001)

[ES3] L. Erdős, J. P. Solovej, Ground state energy of large atoms in a self-generated magnetic field. Commun. Math. Phys. 294, No. 1, 229-249 (2009)

[EFS2] L. Erdös, S. Fournais, J.P. Solovej: Second order semiclassics with self-generated magnetic fields. Preprint: arxiv.org/1105.0512

[EFS3] L. Erdős, S. Fournais, J.P. Solovej: Scott correction for large molecules with a selfgenerated magnetic field. Preprint: arxiv.org/1105.0521

[FS] C. Fefferman and L.A. Seco: On the energy of a large atom, Bull. AMS 23, 2, 525-530 (1990).

[Fo] V. Fock, Bemerkung zur Quantelung des harmonischen Oszillators im Magnetfeld, Z. Physik 47, 446-448 (1928).

[FLW] R. L. Frank, M. Loss, and T. Weidl, Pólya's conjecture in the presence of a constant magnetic field, J. Eur. Math. Soc. 11, 1365-1383, (2009) 
[FLL] J. Fröhlich, E. H. Lieb, and M. Loss: Stability of Coulomb systems with magnetic fields. I. The one-electron atom. Commun. Math. Phys. 104 251-270 (1986)

[H] W. Hughes: An atomic energy bound that gives Scott's correction, Adv. Math. 79, 213-270 (1990).

[Iv1] V.I. Ivrii: Asymptotics of the ground state energy of heavy molecules in a strong magnetic field. I. and II. Russian J. Math. Phys. 4 (1996), no. 1, 29-74 ibid. 5, no. 3, 321-354 (1998).

[Iv2] V.I. Ivrii: Heavy molecules in the strong magnetic field. Russian J. Math. Phys. 4 (1996), no. 4, 449-455.

[Iv3] V.I. Ivrii: Local trace asymptotics in the self-generated magnetic field., arXiv:1108.4188

[IS] V.I. Ivrii and I.M. Sigal: Asymptotics of the ground state energies of large Coulomb systems, Ann. of Math. (2), 138, 243-335 (1993).

[L] E. H. Lieb: Thomas-Fermi and related theories of atoms and molecules, Rev. Mod. Phys. 65. No. 4, 603-641 (1981)

[LL] E. H. Lieb, M. Loss: Stability of Coulomb systems with magnetic fields II. Commun. Math. Phys, 104 271-282 (1986)

[LLS] E. H. Lieb, M. Loss and J. P. Solovej: Stability of Matter in Magnetic Fields, Phys. Rev. Lett. 75, 985-989 (1995)

[LS] E. H. Lieb and B. Simon: The Thomas-Fermi theory of atoms, molecules and solids, Adv. Math. 23, 22-116 (1977)

[LSY1] E. H. Lieb, J. P. Solovej and J. Yngvason: Asymptotics of heavy atoms in high magnetic fields: I. Lowest Landau band region, Commun. Pure Appl. Math. 47, 513-591 (1994)

[LSY2] E. H. Lieb, J. P. Solovej and J. Yngvason: Asymptotics of heavy atoms in high magnetic fields: II. Semiclassical regions. Commun. Math. Phys. 161, 77-124 (1994)

[LT] E. H. Lieb and W. E. Thirring, Bound for the kinetic energy of fermions which proves the stability of matter, Phys. Rev. Lett. 35, 687-689 (1975).

[LY] M. Loss and H.-T. Yau, Stability of Coulomb systems with magnetic fields: III. Zero energy bound states of the Pauli operator. Commun. Math. Phys. 104 (1986), 283290. 
[SW1] H. Siedentop and R. Weikard: On the leading energy correction for the statistical model of an atom: interacting case, Commun. Math. Phys. 112, 471-490 (1987)

[SW2] H. Siedentop and R. Weikard: On the leading correction of the Thomas-Fermi model: lower bound, Invent. Math. 97, 159-193 (1990)

[SW3] H. Siedentop and R. Weikard: A new phase space localization technique with application to the sum of negative eigenvalues of Schrödinger operators, Ann. Sci. Ecole Norm. Sup. (4), 24, no. 2, 215-225 (1991).

[S] B. Simon, Functional Integration and Quantum Physics, Academic Press, 1979

[Sob1] A. V. Sobolev: The quasi-classical asymptotics of local Riesz means for the Schrdinger operator in a strong homogeneous magnetic field. Duke Math. J. 74 (1994), no. 2, 319-429.

[Sob2] A. V. Sobolev: Discrete spectrum asymptotics for the Schrödinger operator with a singular potential and a magnetic field, Rev. Math. Phys 8 (1996) no. 6, 861-903.

[Sob3] A. V. Sobolev: Two-term asymptotics for the sum of eigenvalues of the Schrdinger operator with Coulomb singularities in a homogeneous magnetic field. Asymptotic Anal. 13 (1996), no. 4, 393-421.

[SS] J. P. Solovej, W. Spitzer: A new coherent states approach to semiclassics which gives Scott's correction. Comm. Math. Phys. 241 (2003), no. 2-3, 383-420.

[SSS] J. P. Solovej, T.Ø. Sørensen, W. Spitzer: Relativistic Scott correction for atoms and molecules. Comm. Pure Appl. Math. Vol. LXIII. 39-118 (2010).

[Y] J. Yngvason: Thomas-Fermi theory for matter in a magnetic field as a limit of quantum mechanics. Lett. Math. Phys. 22 (1991), no. 2, 107-117. 